\title{
Radiation Therapy Improves Survival Outcome in Pancreatic Adenocarcinoma: Comparison of a 15-Year Institutional Experience at the University of Nebraska Medical Center with SEER Data
}

\author{
Michael J. Baine ${ }^{1}$ and Chi Lin ${ }^{2}$ \\ ${ }^{1}$ College of Medicine, University of Nebraska Medical Center, Omaha, NE 68198-7521, USA \\ ${ }^{2}$ Department of Radiation Oncology, University of Nebraska Medical Center, Omaha, NE 68198-7521, USA
}

Correspondence should be addressed to Chi Lin; clin@unmc.edu

Received 11 September 2013; Accepted 29 October 2013; Published 23 January 2014

Academic Editor: Oliver Micke

Copyright (C) 2014 M. J. Baine and C. Lin. This is an open access article distributed under the Creative Commons Attribution License, which permits unrestricted use, distribution, and reproduction in any medium, provided the original work is properly cited.

\begin{abstract}
Objectives. We examined the role of radiation therapy (RT) in pancreatic adenocarcinoma (PA) treatment through a 15-year retrospective analysis of patients treated at University of Nebraska Medical Center (UNMC) as well as those from the SEER database. Methods. A total of 561 patients diagnosed with PA at UNMC between 1995 and 2011 and 60,587 patients diagnosed between 1995 and 2009 from the SEER were included. Examined prognostic factors for overall survival (OS) were age, gender, race, stage, year of diagnosis, and treatment with surgery, chemotherapy (CT), or RT. Time to death was plotted by Kaplan-Meier method. A Cox proportional hazards model was used to evaluate prognostic factors for OS. Results. The median OS was 7.3 and 5 months for patients from UNMC and the SEER database, respectively. A Cox model of patients from UNMC showed that RT was associated with improved OS (HR 0.77, $P=0.018$ ) after adjusting for factors including age, race, gender, stage, year of diagnosis, having surgery, or having CT. Cox analysis of patients from the SEER showed similar results (HR $0.65, P<0.0001$ ). Conclusions. RT confers an independent survival advantage in patients being treated for PA which is apparent both at UNMC and through SEER data.
\end{abstract}

\section{Introduction}

Pancreatic adenocarcinoma (PA) is currently the most lethal common cancer worldwide, with a 5-year survival rate of a dismal $6 \%$ [1]. It remains the 4th leading cause of cancer death in the United States and its incidence has been slowly increasing over the decades to its current lifetime risk of approximately 1 in 71 . Further, the prospects for a curative treatment have not been promising. Studies regarding the best therapy for patient with PA have shown conflicting results. As such, there is much controversy regarding optimal treatment regimens for each PA stage [2]. No current consensus exists regarding the use of neoadjuvant or adjuvant therapies or what such therapies should consist of in resectable disease, nor is there widespread agreement on optimal regimen of treatment in borderline resectable or locally advanced unresectable PA. Even more controversial, however, is the role of each treatment modality in each of the proposed therapeutic schemas. While the ultimate goal of PA therapy remains R0 resection, the optimality of the use of chemotherapy (CT), radiation therapy (RT), and chemoradiotherapy (CRT) as well as the timing of their utilization(s) is unknown. Of these, the most controversial aspect of PA treatment is the incorporation of RT or CRT at all [3].

Though RT is limited by its localized nature, especially in a disease well known for early distant spread such as PA, the potential exists for it to be therapeutically indispensable. Local recurrence comprises a significant aspect of treatment failure in $50-75 \%$ of PA patients [3]. Thus, RT has the theoretical capability of reducing the rate of local failure by either (1) shrinking the tumor and thereby allowing for wider 


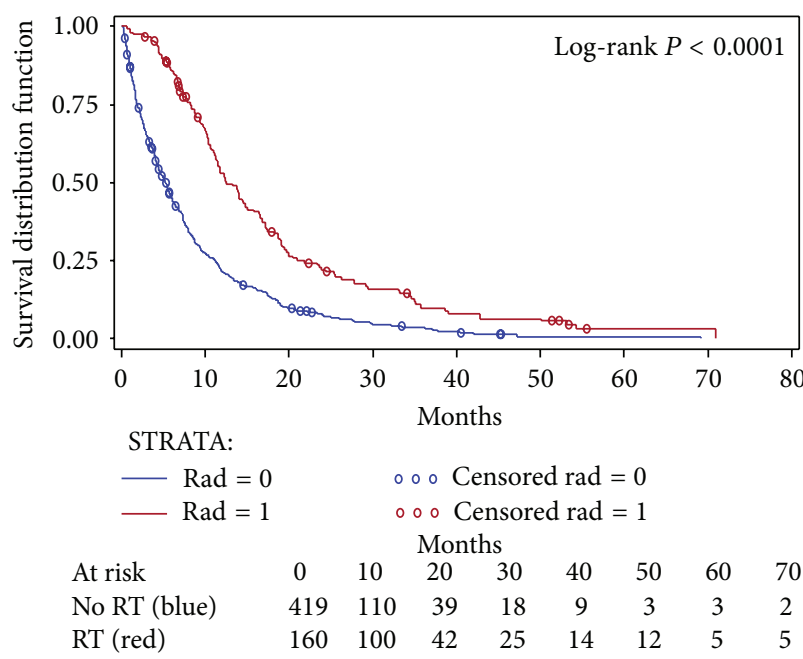

(a)

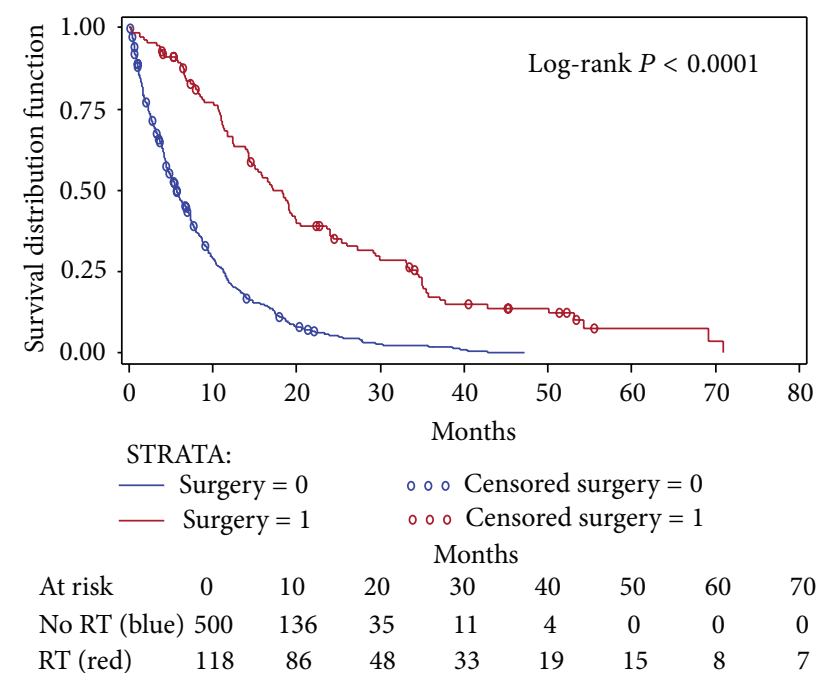

(b)

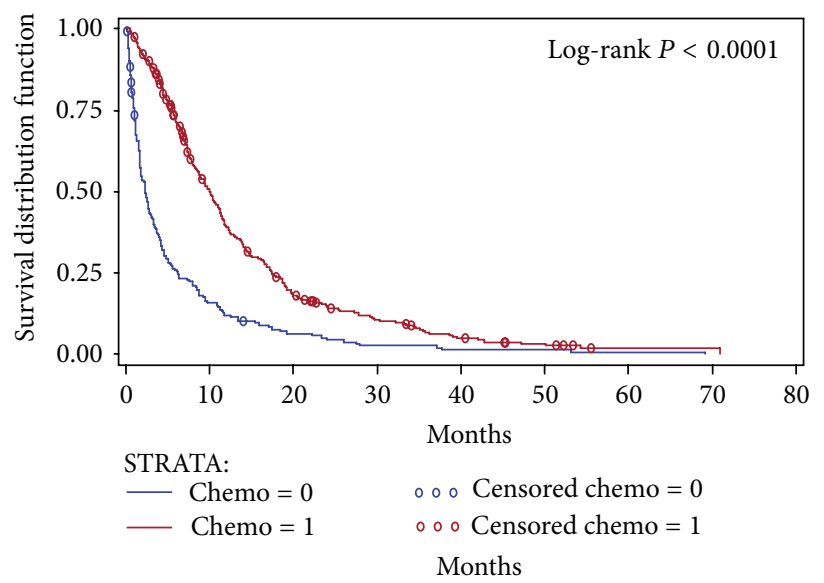

$\begin{array}{lccccccrr}\text { At risk } & 0 & 10 & 20 & 30 & 40 & 50 & 60 & 70 \\ \text { No RT (blue) } & 174 & 28 & 12 & 6 & 4 & 4 & 3 & 2 \\ \text { RT (red) } & 394 & 186 & 68 & 37 & 19 & 11 & 5 & 5\end{array}$

(c)

FIGURE 1: Kaplan-Meier curves of overall survival for PA patients treated at UNMC with (a) radiation therapy, (b) surgery, and (c) chemotherapy. All three treatment modalities independently yield significant improvements in overall survival.

margins of resection or (2) by killing the microscopic foci of residual disease unwittingly left after resection. Further, RT may allow for downstaging of the tumor in borderline resectable disease as well as help to identify which patients have a highly aggressive disease and thus are unlikely to derive benefit from resection [3]. It is for these purposes that many centers in the United States offer RT or CRT as neoadjuvant, adjuvant, or primary therapy for all PA patients without metastatic disease [2]. However, opponents of this strategy cite the fact that both RT and CRT show minimal efficacy in preclinical and clinical data and thus neoadjuvant RT/CRT may allow resectable patients to advance to unresectability, while adjuvant RT/CRT creates greater risk of toxicity as compared to CT alone without proven benefit. This argument has gained a significant following in Europe and is the main thrust for European PA treatment regimens to forgo RT completely in lieu of adjuvant CT alone [2].
In an attempt to further clarify the role and benefit of RT in PA treatment, comprehensive data from all PA patients treated at the University of Nebraska Medical Center (UNMC) from 1995 to 2011 were retrospectively analyzed. Further, this data was then compared to US national data derived from the National Cancer Institute's Surveillance, Epidemiology, and End Results (SEER) database to determine epidemiologic efficacy of PA RT as well as how well local and national data parallel each other.

\section{Materials and Methods}

2.1. UNMC Patient Data. A patient record search from 1995 to 2011 identified a total of 619 patients who were treated for PA at the University of Nebraska Medical Center. After excluding missing data, there were 561 patients. Data including patient's age at diagnosis $(\leq 68 />68)$, gender, race 


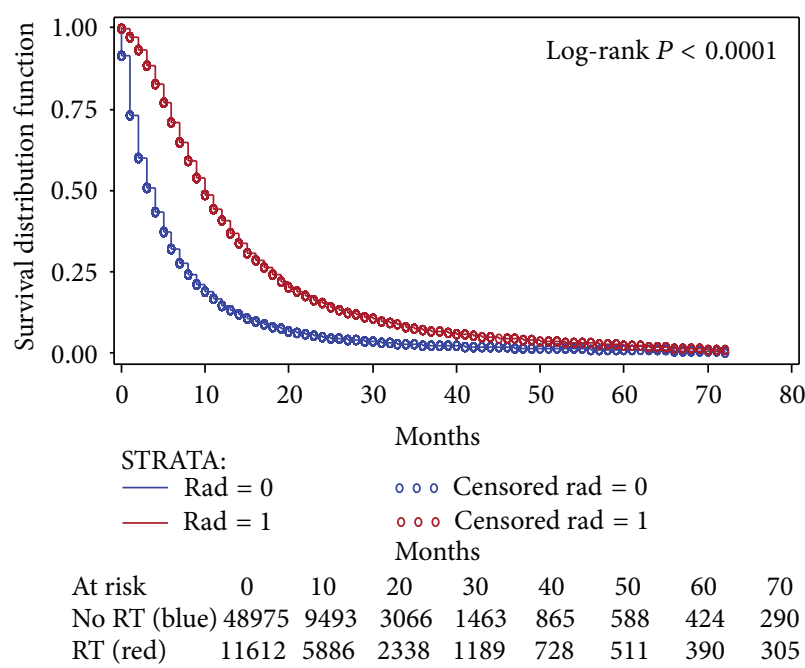

(a)

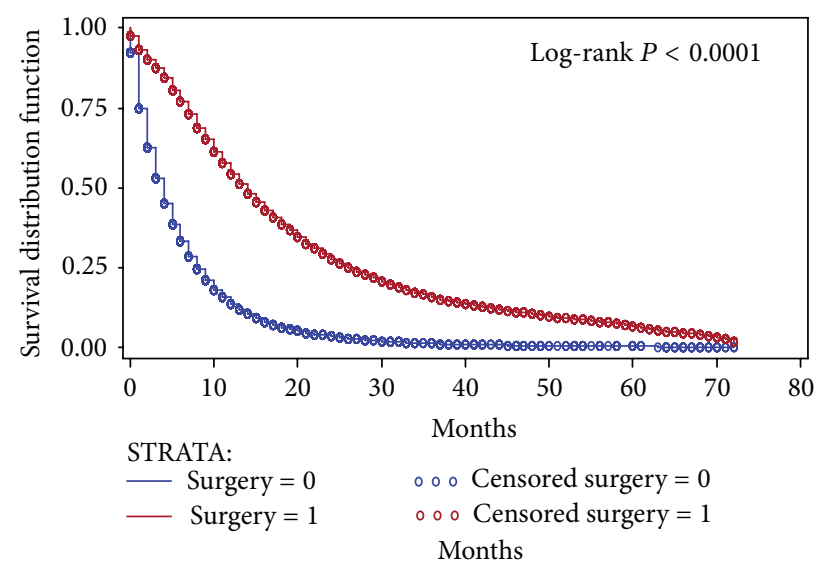

$\begin{array}{lllllllll}\text { At risk } & 0 & 10 & 20 & 30 & 40 & 50 & 60 & 70\end{array}$

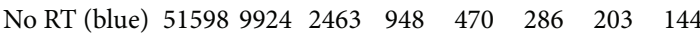
$\begin{array}{lllllllll}\text { RT (red) } & 8989 & 5455 & 2941 & 1704 & 1123 & 813 & 611 & 451\end{array}$

(b)

FIGURE 2: Kaplan-Meier curves of overall survival for PA patient data derived from the SEER database following treatment with (a) radiation therapy and (b) surgery. Both treatment modalities independently yield significant improvements in overall survival.

(white or nonwhite), AJCC tumor stage (I, II, III, or IV), year of diagnosis (1995-1999/2000-2003/2004-2006/20072011), if the patient underwent surgical resection, CT, or RT, and current status of either living or dead were obtained. Time of survival was determined from time at diagnosis until confirmed date of death or date of last encounter. All aspects of this retrospective study were preauthorized by the UNMC Internal Review Board.

2.2. SEER Data. A total of 65,587 patients were identified from the SEER database who were diagnosed with PA from 1995 to 2009. After excluding missing data, there were 60587 patients. Data including patient age at diagnosis $(\leq 68 />68)$, gender, race (white or nonwhite), tumor stage (local, regional, or distant), year of diagnosis (1995-1999/2000-2003/20042006/2007-2009), type of treatment (RT/surgery), and current status of being either living or dead were obtained. As SEER data does not include information on CT, no CT data could be utilized in this analysis. All data was obtained from the National Cancer Institute's SEER website (http://seer.cancer.gov/) with permission.

2.3. Statistical Analysis. Chi-square tests were used to compare the difference in proportions for baseline patient characteristics between UNMC and SEER databases. Time to event distributions were estimated using the Kaplan-Meier method and were compared using the log-rank test. A Cox proportional hazards model was used to evaluate prognostic factors for overall survival. Data were analyzed using the SAS 9.3 statistical software (Cary, NC, USA).

\section{Results}

3.1. Demographic Data. The median age was 68 and 69 and median OS was 7.3 and 5 months for patients from UNMC and the SEER database, respectively. UNMC patients were more likely to be white ( $92 \%$ versus $81 \%, P=0.02$ ), receiving RT (32\% versus $19 \%, P=0.04)$, and diagnosed more recently $(P=0.03)$ than patients from the SEER database. Other patient characteristics such as age, gender, and stage are similar between these two databases. Complete demographic information can be found in Table 1 .

3.2. Radiation Therapy Is Associated with Improved Overall Survival in PA Patients Treated at UNMC. Overall survival analysis revealed that patients who received RT had a significantly greater median survival rate than those who did not receive RT (13.8 versus 5.3 months, log-rank $P<$ 0.0001 , Figure 1(a), Table 2). Similarly, surgery was associated with the greatest increase in median overall survival at 13.0 months (18.7 versus 5.7 months, log-rank $P<$ 0.0001 , Figure 1(b), Supplementary Table 1 available online at http://dx.doi.org/10.1155/2014/708317), while CT was associated with the least increase in median overall survival at 7.9 months (10.2 versus 2.3 months, $P<0.0001$, Figure 1(c), See Supplementary Table 2). Cox multivariate analyses of patient data from UNMC indicate that RT, CT, surgery, tumor stage, and patient age are independent prognostic factors in PA, while race, gender, and year of diagnosis were not found to be associated with overall survival (Table 3 ).

3.3. Radiation Therapy Is Associated with Improved Overall Survival in PA Patients Included in SEER Database. Similarly, both RT and surgery were found to independently improve median survival of PA patients by 7 months (11 versus 4 months, log-rank $P<0.0001)$ and 9 months (15 versus 4 months, $\log$-rank $P<0.0001$ ), respectively (Figure 2, Table 2 and Supplementary Table 1). Cox analysis revealed that RT, surgery, tumor stage, and patient age are all independent prognostic factors of patient overall survival among SEER PA 
TABLE 1: Demographics.

\begin{tabular}{|c|c|c|c|}
\hline & UNMC & SEER & $P\left(\chi^{2}\right)^{\mathrm{c}}$ \\
\hline$n$ & 561 & 60587 & \\
\hline \multicolumn{4}{|l|}{ Age $n(\%)$} \\
\hline$\leq 68$ & $278(49.55 \%)$ & $28924(47.74 \%)$ & \multirow{2}{*}{0.8} \\
\hline$>68$ & $383(50.45 \%)$ & $31663(52.26 \%)$ & \\
\hline \multicolumn{4}{|l|}{ Gender $n(\%)$} \\
\hline Male & $321(57.22 \%)$ & $29599(48.85 \%)$ & \multirow{2}{*}{0.2} \\
\hline Female & $240(42.78 \%)$ & $30988(51.15 \%)$ & \\
\hline \multicolumn{4}{|l|}{ Race $n(\%)$} \\
\hline White & $516(91.98 \%)$ & $49089(81.02 \%)$ & \multirow{2}{*}{0.02} \\
\hline Nonwhite & $45(8.02 \%)$ & $11498(18.98 \%)$ & \\
\hline \multicolumn{4}{|l|}{ Stage $n(\%)$} \\
\hline $\mathrm{I}$ & $48(8.56 \%)$ & & \multirow{7}{*}{$0.1^{\mathrm{b}}$} \\
\hline II & $150(26.74 \%)$ & & \\
\hline III & $79(14.08 \%)$ & & \\
\hline IV & $284(50.62 \%)$ & & \\
\hline Local & & $4384(7.24 \%)$ & \\
\hline Regional & & $18782(31.00 \%)$ & \\
\hline Distant & & $37421(61.76 \%)$ & \\
\hline \multicolumn{4}{|l|}{ Surgery $n(\%)$} \\
\hline No & $446(79.50 \%)$ & $51598(85.16 \%)$ & \multirow{2}{*}{0.29} \\
\hline Yes & $115(20.50 \%)$ & $8989(14.84 \%)$ & \\
\hline \multicolumn{4}{|l|}{ Radiation $n(\%)$} \\
\hline No & $383(68.27 \%)$ & $48975(80.83 \%)$ & \multirow{2}{*}{0.04} \\
\hline Yes & $178(31.73 \%)$ & $11612(19.17 \%)$ & \\
\hline \multicolumn{4}{|l|}{ Chemotherapy $n$ (\%) } \\
\hline No & $151(26.92 \%)$ & & \\
\hline Yes & $410(73.08 \%)$ & & \\
\hline \multicolumn{4}{|c|}{ Year of diagnosis $n(\%)$} \\
\hline 1995-1999 & $129(22.99 \%)$ & $9628(15.89 \%)$ & \multirow{4}{*}{0.03} \\
\hline $2000-2003$ & $94(16.76 \%)$ & $17950(29.63 \%)$ & \\
\hline 2004-2006 & $86(15.33 \%)$ & $15513(25.60 \%)$ & \\
\hline $2007-2011 / 2009^{a}$ & $252(44.92 \%)$ & $17496(28.88 \%)$ & \\
\hline \multicolumn{4}{|l|}{ Living $n(\%)$} \\
\hline Yes & $51(9.09 \%)$ & $5533(9.13 \%)$ & \multirow{2}{*}{0.8} \\
\hline No & $510(90.91 \%)$ & 55054 (90.87\%) & \\
\hline
\end{tabular}

${ }^{\mathrm{a}}$ UNMC Data extended to 2011 to enhance patient numbers.

${ }^{\mathrm{b}}$ Nonstage IV was grouped together and was compared to stage IV.

${ }^{\mathrm{c}}$ Percentage of each category was compared.

TABLe 2: Median overall survival (95\% CI) with and without radiation therapy.

\begin{tabular}{lcc}
\hline & RT & No RT \\
\hline UNMC & $13.8(11.4-15.8)$ & $5.3(4.4-6.1)$ \\
SEER & $11(10-11)$ & 4 \\
\hline
\end{tabular}

OR $=0.94795 \%$ CI $0.2069-4.3325$.

CI: confidence interval; RT: radiation therapy; OR: odds ratio.

patients. In this extended patient population, however, race and gender were found to be independently associated with overall survival as well (Table 3 ).
3.4. Comparison of PA Patient Survival, UNMC versus National SEER Data. No significant difference was found between the UNMC and SEER data for the survival advantages conferred by either surgery or RT. The odds ratio (OR) and $95 \%$ confidence interval (CI) for the surgical survival advantage were found to be 0.874 and $0.2057-3.7213$, respectively, between the databases. For the RT-conferred survival advantage, the OR ( $95 \% \mathrm{CI})$ between the two databases was found to be 0.947 (0.2069-4.3325).

\section{Discussion}

The inclusion of RT in the treatment of PA continues to be a source of contention. While the addition of RT has several theoretical advantages, such as improved local control postresection, preoperative tumor shrinkage, and identification of patients with highly aggressive tumors who are unlikely to significantly benefit from resection, none have been consistently demonstrated clinically [3]. Further, standard PA irradiation consists of 50.4 Gy delivered in 1.8$2.0 \mathrm{~Gy}$ fractions and thus takes at least 5 weeks to complete, a significant burden for patients whose life expectancy is rarely greater than 1 year [2]. This conflict between the speculative and intermittently demonstrable advantages provided by RT and its lack of clear benefit paired with its high cost to the patient has culminated into an inconsistency in therapeutic regimens offered to PA patients both on national and international levels.

Unfortunately, clinical trial data do little to sway the arguments of this controversy. Currently, no studies have been conducted to determine the efficacy of neoadjuvant RT or CRT relative to adjuvant treatment or direct surgical resection. Several trials, as well as numerous single center studies, have however been completed investigating the role of adjuvant CRT versus CT or observation in PA. The Gastrointestinal Tumor Study Group (GITSG) has reported a statistically significant 2.5 -month increase in survival for patients treated with CRT versus CT alone, while the European Organization for Research and Treatment of Cancer showed a nonsignificant trend toward survival improvement for adjuvant CRT as compared to observation $[4,5]$. These trials, however, have been met with much criticism due to suboptimal radiation dosing, use of split-course radiation, and low patient numbers in the GITSG trial [6]. More recently, the Eastern Cooperative Oncology Group (ECOG) also showed a 1.8-month survival advantage to CRT as compared to CT alone that was found to be statistically significant [7]. Further, two prospective institutional studies from Johns Hopkins as well as a retrospective study using combined data from Johns Hopkins and Mayo Clinic show consistent survival advantages of CRT as compared to observation that are highly statistically significant [8-10]. Conversely, the largest trial investigating the role of adjuvant RT/CRT in PA was conducted by the European Study Group for Pancreatic Cancer (ESPAC-1) which showed a significant survival disadvantage in its CRT arm as compared to both $\mathrm{CT}$ and observation [11]. These results were further bolstered by a Federation Francophone de Cancerologie Digestive and 
TABLE 3: Cox multivariate analysis of overall survival.

\begin{tabular}{|c|c|c|c|c|}
\hline & UNMC hazard ratio $(95 \% \mathrm{CI})$ & $P\left(\chi^{2}\right)$ & SEER hazard ratio $(95 \% \mathrm{CI})$ & $P\left(\chi^{2}\right)$ \\
\hline Radiation therapy (yes versus no) & $0.772(0.623-0.957)$ & 0.018 & $0.645(0.631-0.660)$ & $<0.0001$ \\
\hline Chemotherapy (yes versus no) & $0.424(0.343-0.523)$ & $<0.0001$ & & \\
\hline Surgery (yes versus no) & $0.353(0.268-0.466)$ & $<0.0001$ & $0.460(0.448-0.473)$ & $<0.0001$ \\
\hline Stage (advanced versus early) & $1.236(1.113-1.372)$ & $<0.0001$ & $1.425(1.403-1.447)$ & $<0.0001$ \\
\hline Age $(>68$ versus $\leq 68)$ & $1.371(1.142-1.645)$ & 0.001 & $1.007(1.006-1.007)$ & $<0.0001$ \\
\hline Race (nonwhite versus white) & $0.723(0.514-1.016)$ & 0.062 & $1.040(1.018-1.063)$ & 0.0003 \\
\hline Gender (male versus female) & $1.066(0.892-1.274)$ & 0.481 & $1.042(1.025-1.060)$ & $<0.0001$ \\
\hline Year (earlier versus current) & $1.014(0.996-1.033)$ & 0.128 & $1.067(1.059-1.076)$ & $<0.0001$ \\
\hline
\end{tabular}

Societe Francaise de Radiotherapie Oncologique, (FFCDSFRO) study in which a statistically significant 4.4-month survival disadvantage was observed in the CRT versus CT group [12].

In an attempt to further elucidate the role of RT in PA treatment, we undertook a 15 -year retrospective study including institutional data from the University of Nebraska Medical Center as well as national data from the SEER database to determine the independent survival advantage offered by RT in PA patients. In total, data from 561 patients treated at UNMC as well as 60,587patients included in the SEER database diagnosed with PA between 1995 and 2011 were analyzed and demonstrated a clear improvement in overall survival associated with RT administration when corrected for patient factors such as age, tumor stage, and utilization of CT or surgical resection.

The impact of these results is further substantiated through their comparison to those published in past studies. In a previously published institutional experience, consisting of a 10-year retrospective analysis of patients treated at Loma Linda University between 1998 and 2009, it was shown that patients treated with RT had a median overall survival of 12.5 months versus 9.1 months in those treated with CT alone but that this improvement was not statistically significant $(P=0.998)[13]$. Primary arguments against the results of this institutional study, however, include its low patient numbers (116 patients total with only 32 patients not receiving RT) as well as its lack of inclusion of resectable or borderline resectable patients which represent key populations for whom RT can have significant therapeutic impact. Additionally, RT has been epidemiologically investigated in PA patients using SEER data through multiple previous studies, though none sought to directly contrast the impact of RT on overall survival from that conferred by alternative treatment regimens or nontreatment patient factors in such an extensive PA patient population. A prime example of this includes two studies by Opfermann et al. and Artinyan et al., respectively, in which it was similarly found that the addition of RT significantly increases overall survival in PA (5-month overall survival improvement, $P<0.001$ in both studies) as compared to surgery alone, though they limited their investigations to patients who successfully underwent surgical resection and had known lymph node status thus reducing their patient populations to 3314 and 1930, respectively $[14,15]$. An additional study by McDade et al. also demonstrated a survival advantage with RT administration in PA using SEER data, though they limited its analysis to adjuvant RT only [16]. Two further studies have investigated the impact of adjuvant versus neoadjuvant $\mathrm{RT}$ in resected PA, each independently discovering that both neoadjuvant and adjuvant RT confer similar survival advantages over nonirradiated patients, though neither compared these results either individually or combined to overall survivals seen with alternate therapeutic strategies $[17,18]$. In contrast, a study by Stessin et al. indicated that neoadjuvant RT is associated with a significantly greater survival advantage over surgery alone than is adjuvant RT, with the source of noncongruence between these studies being currently unclear [19].

Despite its novelty and strength of a large patient population, the current study has multiple drawbacks. First, no data was analyzed regarding the effect of RT on patient performance status, quality of life, or quantitative or qualitative toxicities suffered; all subjects considered highly important in a malignancy with near uniform fatality such as PA. Second, neither progression-free survival nor cancer-specific survival was investigated in the present study though they may prove of substantial influence in the consideration of RT's true therapeutic utility. Further this study made no corrections for radiation dosing, treatment method, or deviation from treatment plan, all of which could impact RT efficacy as well as patient treatment tolerability. These shortcomings necessitate further study into the influence of RT in PA to definitely determine the true utility and clinically relevant survival impact this promising though contentious treatment modality offers.

Results of this study serve to add credence to the addition of RT to PA treatment regimens. Though PA remains generally considered a treatment-refractory malignancy, increase in life expectancy is independently evident with each of the three treatment modalities available, including surgery, CT, and RT, and their utilization should be offered to the fullest extent tolerated by the patient. Further, as novel methods for PA RT delivery such as stereotactic body RT and intensity modulated RT become more widely available, the treatment toxicity will be reduced [2]. While additional studies are necessary to prove the clinical efficacy of these innovative RT techniques in PA, their application promises to further diminish future arguments against RT administration in PA patients and more clearly cement the role of RT as a staple in PA therapy. 
In conclusion, our results indicate that RT confers an independent survival advantage in patients being treated for PA which is apparent through both Nebraska and SEER data. These results extend available evidence indicating that RT has a valid role in the treatment of PA and further supports its widespread use as an accepted PA therapeutic modality.

\section{Disclosure}

The abstract of this paper has been accepted to the 55th ASTRO annual meeting for presentation in the poster session.

\section{Conflict of Interests}

The authors declare that there is no conflict of interests regarding the publication of this paper.

\section{References}

[1] Cancer Facts \& Figures, American Cancer Society, Atlanta, Ga, USA, 2013.

[2] K. A. Goodman and C. Hajj, "Role of radiation therapy in the management of pancreatic cancer," Journal of Surgical Oncology, vol. 107, no. 1, pp. 86-96, 2013.

[3] F. Wang and P. Kumar, "The role of radiotherapy in management of pancreatic cancer," The Journal of Gastrointestinal Oncology, vol. 2, pp. 157-167, 2011.

[4] "Treatment of locally unresectable carcinoma of the pancreas: comparison of combined-modality therapy (chemotherapy plus radiotherapy) to chemotherapy alone. Gastrointestinal Tumor Study Group," Journal of the National Cancer Institute, vol. 80, no. 10, pp. 751-755, 1988.

[5] J. H. Klinkenbijl, J. Jeekel, T. Sahmoud et al., "Adjuvant radiotherapy and 5-fluorouracil after curative resection of cancer of the pancreas and periampullary region: phase III trial of the EORTC Gastrointestinal Tract Cancer Cooperative Group," Annals of Surgery, vol. 230, no. 6, pp. 776-784, 1999.

[6] M. W. Saif, "Advancements in the management of pancreatic cancer: 2013," Journal of the Pancreas, vol. 14, no. 2, pp. 112-118, 2013.

[7] D. J. Klaassen, J. M. MacIntyre, G. E. Catton, P. F. Engstrom, and C. G. Moertel, "Treatment of locally unresectable cancer of the stomach and pancreas: a randomized comparison of 5fluorouracil alone with radiation plus concurrent and maintenance 5-fluorouracil-an Eastern Cooperative Oncology Group study," Journal of Clinical Oncology, vol. 3, no. 3, pp. 373-378, 1985.

[8] C. J. Yeo, R. A. Abrams, L. B. Grochow et al., "Pancreaticoduodenectomy for pancreatic adenocarcinoma: postoperative adjuvant chemoradiation improves survival: a prospective, single-institution experience," Annals of Surgery, vol. 225, no. 5, pp. 621-636, 1997.

[9] J. M. Herman, M. J. Swartz, C. C. Hsu et al., "Analysis of fluorouracil-based adjuvant chemotherapy and radiation after pancreaticoduodenectomy for ductal adenocarcinoma of the pancreas: results of a large, prospectively collected database at the Johns Hopkins Hospital," Journal of Clinical Oncology, vol. 26, no. 21, pp. 3503-3510, 2008.

[10] C. C. Hsu, J. M. Herman, M. M. Corsini et al., "Adjuvant chemoradiation for pancreatic adenocarcinoma: the Johns
Hopkins Hospital-Mayo Clinic collaborative study," Annals of Surgical Oncology, vol. 17, no. 4, pp. 981-990, 2010.

[11] W. F. Regine, K. A. Winter, R. A. Abrams et al., "Fluorouracil vs gemcitabine chemotherapy before and after fluorouracilbased chemoradiation following resection of pancreatic adenocarcinoma: a randomized controlled trial," The Journal of the American Medical Association, vol. 299, no. 9, pp. 1019-1026, 2008.

[12] B. Chauffert, F. Mornex, F. Bonnetain et al., "Phase III trial comparing intensive induction chemoradiotherapy (60 Gy, infusional 5-FU and intermittent cisplatin) followed by maintenance gemcitabine with gemcitabine alone for locally advanced unresectable pancreatic cancer. Definitive results of the 2000-01 FFCD/SFRO study," Annals of Oncology, vol. 19, no. 9, pp. 15921599,2008

[13] N. K. Malik, K. S. May, R. Chandrasekhar et al., "Treatment of locally advanced unresectable pancreatic cancer: a 10-year experience," The Journal of Gastrointestinal Oncology, vol. 3, no. 4, pp. 326-334, 2012.

[14] K. J. Opfermann, A. E. Wahlquist, E. Garrett-Mayer, R. Shridhar, L. Cannick, and D. T. Marshall, "Adjuvant radiotherapy and lymph node status for pancreatic cancer: results of a study from the Surveillance, Epidemiology, and End Results (SEER) registry data," American Journal of Clinical Oncology, 2012.

[15] A. Artinyan, M. Hellan, P. Mojica-Manosa et al., "Improved survival with adjuvant external-beam radiation therapy in lymph node-negative pancreatic cancer: a United States populationbased assessment," Cancer, vol. 112, no. 1, pp. 34-42, 2008.

[16] T. P. McDade, J. S. Hill, J. P. Simons et al., "A national propensityadjusted analysis of adjuvant radiotherapy in the treatment of resected pancreatic adenocarcinoma," Cancer, vol. 116, no. 13, pp. 3257-3266, 2010.

[17] J. Franko, D. R. Puri, and C. D. Goldman, "Impact of radiation therapy sequence on survival among patients with resected pancreatic head ductal carcinoma," Annals of Surgical Oncology, vol. 19, no. 1, pp. 26-30, 2012.

[18] L. Hazard, J. D. Tward, A. Szabo, and D. C. Shrieve, "Radiation therapy is associated with improved survival in patients with pancreatic adenocarcinoma: results of a study from the Surveillance, Epidemiology, and End Results (SEER) registry data," Cancer, vol. 110, no. 10, pp. 2191-2201, 2007.

[19] A. M. Stessin, J. E. Meyer, and D. L. Sherr, "Neoadjuvant radiation is associated with improved survival in patients with resectable pancreatic cancer: an analysis of data from the Surveillance, Epidemiology, and End Results (SEER) registry," International Journal of Radiation Oncology, Biology, Physics, vol. 72, no. 4, pp. 1128-1133, 2008. 


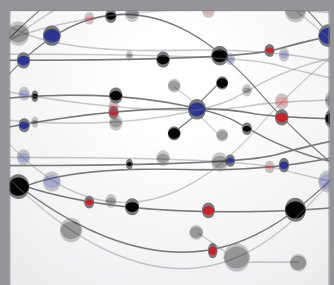

The Scientific World Journal
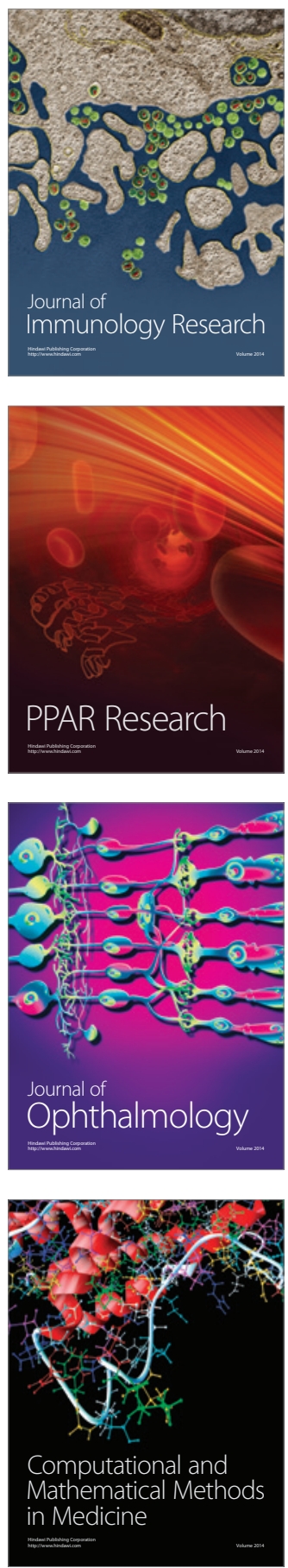

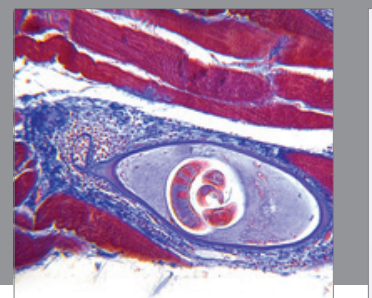

Gastroenterology

Research and Practice
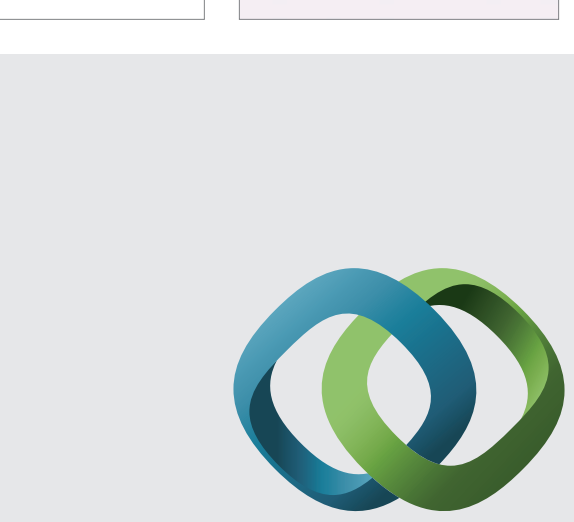

\section{Hindawi}

Submit your manuscripts at

http://www.hindawi.com
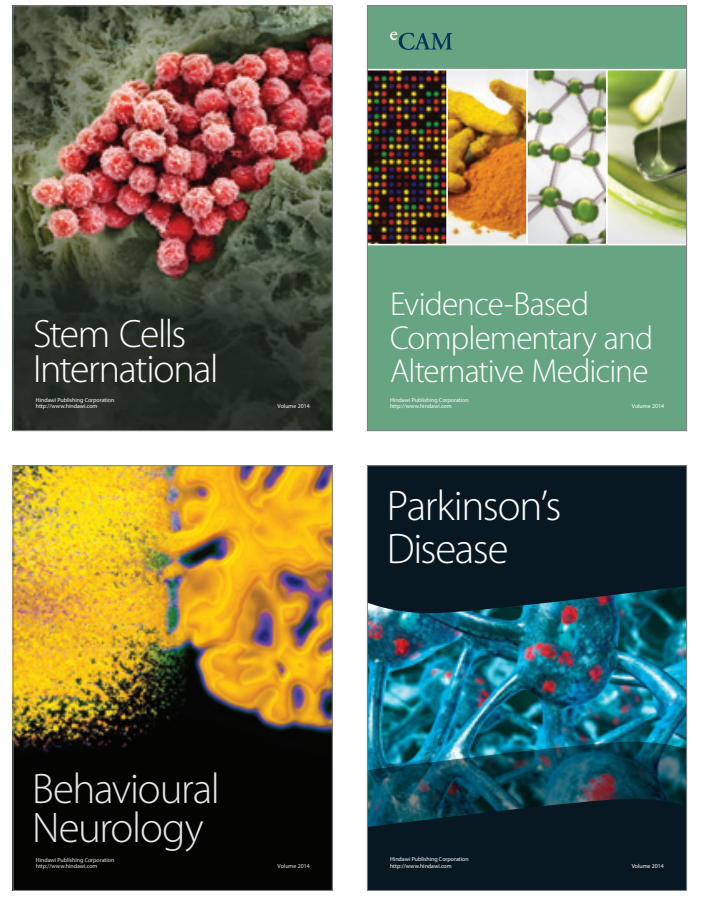
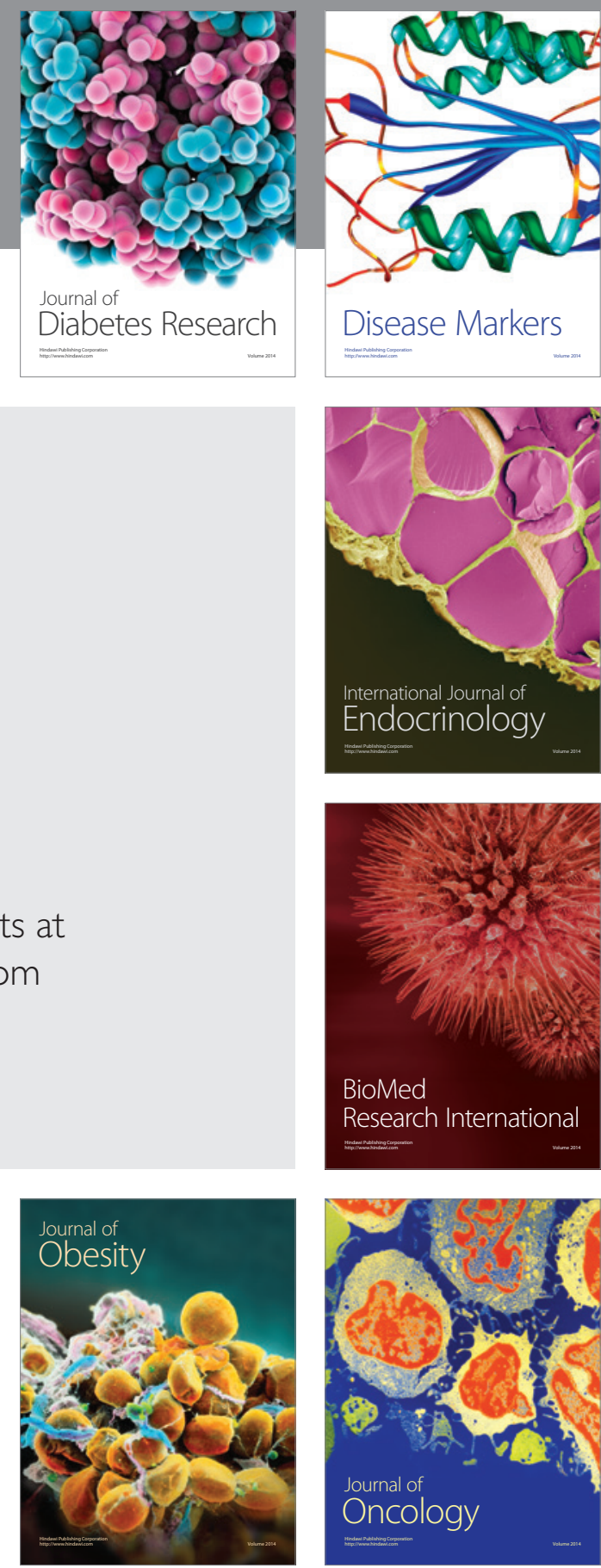

Disease Markers
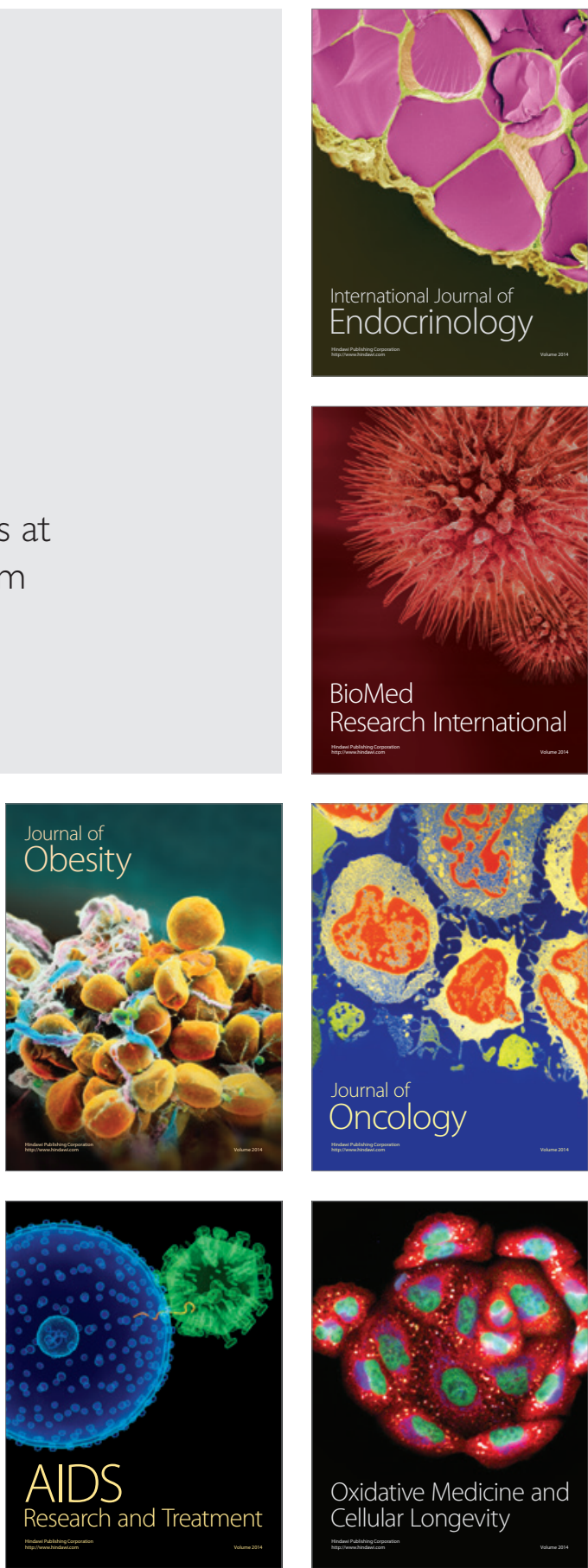